\title{
The Theoretical Investigation of a S-spring in a Suspended Armature Valve
}

\author{
Chuang $\mathrm{Xu}^{1}$, Qiang Wang ${ }^{1}$, Aiqing Huang ${ }^{2}$ and Siwei Miao ${ }^{2}$ \\ ${ }^{1}$ Shanghai Institute of Space Propulsion, Shanghai minhang district wanfang road 801, 201112 Shanghai, China \\ ${ }^{2}$ Research Center of Space Engine, Shanghai minhang district wanfang road 801, 201112 Shanghai, China
}

\begin{abstract}
The development of suspended armature valves is a trend in the future spacecraft propulsion systems. Compared to solenoid valves, the suspended armature valves have no friction surface, which eliminates the sliding interaction and the associated generations of particulate. Besides, the structure is more compact in axis direction for a suspended armature valve than a solenoid valve. On account of certain preferred characteristics, such as a long cycle-life, improvement in seal performance, and superior tolerance to the deformation and mechanical environment, the suspended armature valve mostly adopts S-shaped compression springs (S-spring). It is of significance to design a durable and reliable S-spring. The main objective of the present work is to introduce a theoretical methodology to design the S-spring. This dramatically reduces research time and controls associated cost.
\end{abstract}

Keywords-suspended armature valve; s-spring; theoretical methodology

\section{INTRODUCTION}

The thruster valve is one of the most important parts of spacecraft propulsion systems due to its irreplaceable function in the spacecraft missions. Failure of the valves would have a fatal situation, such as a leakage of the propulsion and even a loss of the spacecraft attitude. Thus, developing reliable and durable thruster valves has become the first priority for an aerospace system ${ }^{[1]}$.

Thruster valves mostly adopt solenoid valves, which have two different structures, namely the sliding fit valve and suspended armature valve. The sliding fit surface interaction will generate metallic product during repeated movement, which would introduce risk and variability. The suspended armature valve eliminates the sliding interaction and the associated generation of particulate. Besides, there are some other positive advantages for the suspended armature valve. On one hand, the sealing of the valve improves because the sealing surface maintains coherence after repeated opening and closing movements. Eliminating the sliding friction and improving service life provide a dramatic improvement over the heritage design. The suspended armature design solution using a S-spring is predicted to offer an unprecedented 30-year successful pedigree ${ }^{[2]}$.

On account of certain preferred characteristics, such as a long cycle-life, improvement in seal performance, and superior tolerance to the deformation and mechanical environment, the suspended armature valve mostly adopts S-shaped compression
springs(S-spring) ${ }^{[3]}$. The armature is supported in the air by two S-shaped compression springs, similar to a leaf spring.

The S-spring is a key part of the suspended armature valve. The outer part of the spring is fixed in the valve body and the inner part is fixed in the armature. A cutting spoke works the leaf spring and maintains the armature in its central position by ensuring stiffness in the radial displacement. Figure I depicts a $150 \mathrm{~N}$-class thruster valve, where a S-spring is located in.

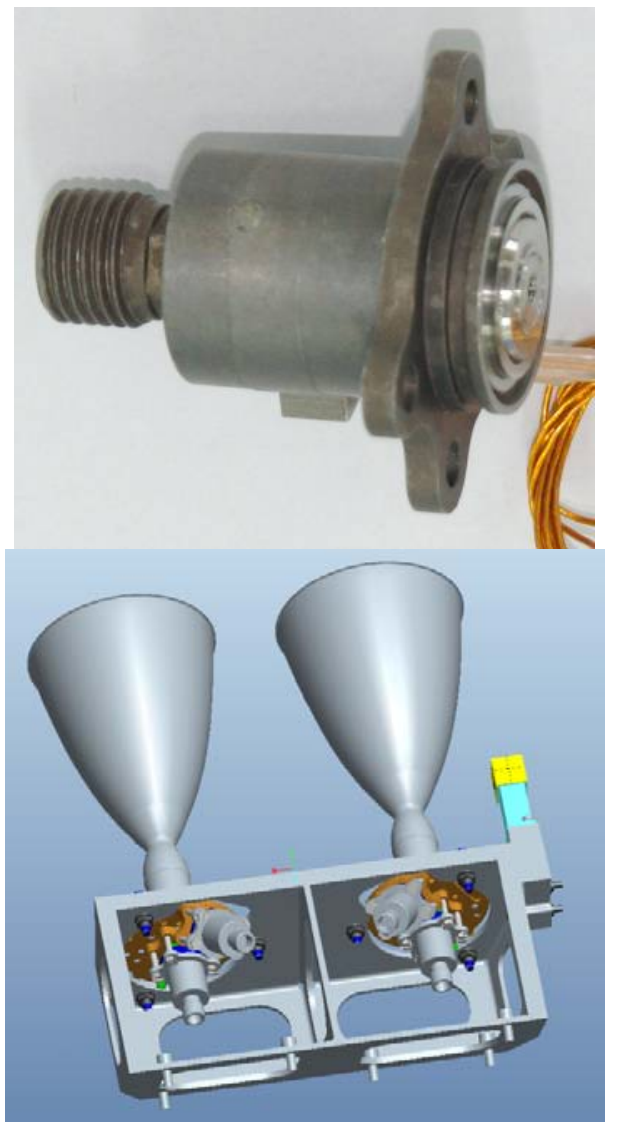

FIGURE I. S-SPRING IN A SUSPENDED ARMATURE VALVE

The challenges in the S-spring design are to determine the wrap size in the limited space of the suspended armature valve, choosing the optimal structure and configuration to balance the necessary spring force and the stress distribution. A S-shaped compression spring is provided in Figure II. In addition to guaranteeing the cycle life and material compatibility, the 
S-spring material should select properly. A cold work hardening stainless steel is selected, which combines the advantages of martensitic stainless steel and austenitic stainless steel.

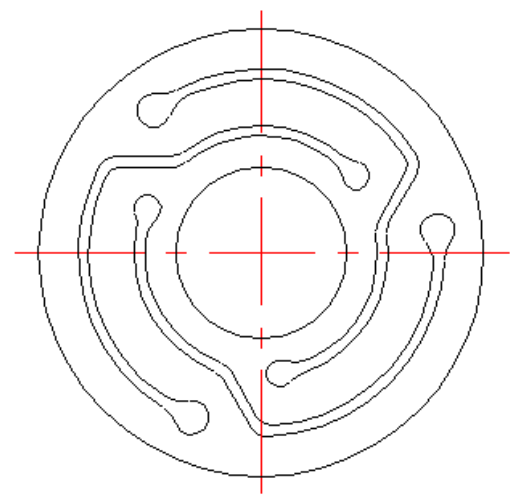

FIGURE II. S-SHAPED COMPRESSION SPRING

Some authors have studied the performance of different S-spring characters, but they haven't provided a method to calculate the parameters of the S-spring. The objective of this paper is to provide a method for the theoretical design of $\mathrm{S}$-springs. This dramatically reduces research time and controls associated cost.

\section{THEORETICAL METHODOLOGY}

Contrast to the coil spring, the S-spring has the advantage of vibration absorption and offset mass deformation. In order to increase the cycle life of the S-spring, it is of significance to design and manufacture accurately. The structure size, thickness and bend curve shape have a big influence on the S-spring performance, which makes a high criterion for the design and manufacture quality.

It is critical that some key parameters should be logically selected in the S-spring design in Figure III, including the outer diameter, thickness, and bend curve shape. The outer diameter is restricted by the limited space of the suspended armature valve. The S-spring with a thin thickness, has allowed the bending stress to maintain appropriate to fit large deformation. It is quite difficult to optimize the bend curve shape to balance the essential stiffness and the reasonable distribution of $\mathrm{S}$-spring stress. At present, there is no existing theoretical formula to calculate the stiffness and the stress of the S-spring. According to the similar characteristic of leaf spring, the S-spring can be regarded as a leaf spring structure supported by $\mathrm{N}$ bend curves. On the basis of curvature radius, each bend curve could be divided in the linearization method. Assuring each bend curve can be divided into $M$ rectangular segments with one end fixed and another end free, each segment would be calculated separately as illustrated bellow.

$$
K_{m}^{\prime}=\frac{3 E}{L_{m}{ }^{3}} \frac{b t^{3}}{12\left(1-\mu^{2}\right)}
$$

Where $\mathrm{K}_{\mathrm{m}}$ is the stiffness of each segment, $\mathrm{E}$ is the Young's Modulus, $\mathrm{b}$ is the width of each segment, $\mathrm{t}$ is the thickness of the S-spring, $L_{m}$ is the length of each segment and $\mu$ is the Poisson's ratio.

Each bend curve is calculated as follows.

$$
K_{n}^{\prime}=\prod_{1}^{M} K_{m}^{\prime} / \sum_{1}^{M} \frac{\prod_{1}^{M} K_{m}^{\prime}}{K_{m}^{\prime}}
$$

Where $\mathrm{K}_{\mathrm{n}}$ is the stiffness of each bend curve.

The S-spring is composed with $\mathrm{N}$ bend curves.

$$
K^{\prime}=\sum_{1}^{n} K_{n}^{\prime}
$$

Where K'is the stiffness of the S-spring.

The maximum stress of each bend curve was obtained.

$$
\sigma_{\max }=\frac{F_{n} L}{Z_{m}}
$$

Where $\sigma_{\max }$ is the maximum stress of the S-spring, $F_{n}$ is the force of the $\mathrm{S}$-spring, $\mathrm{L}$ is the length of the $\mathrm{S}$-spring and $\mathrm{Z}_{\mathrm{m}}$ is the section modulus. (See Figure III)
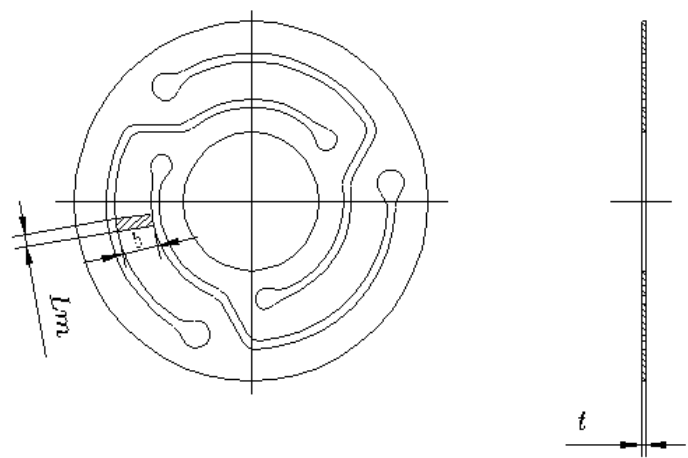

FIGURE III. S-SPRING KEY PARAMETER

As reference to formula (1), the stiffness of the S-spring is proportional to the fist power of the section width and third power of the section thickness, while varies inversely with third power of the curve length.

Depending on the working function of the S-spring, the maximum stress is deduced as follows. 


$$
\begin{aligned}
\sigma_{\max }=\frac{F_{n} L}{Z_{m}}=\frac{\left(F_{t n}+K_{n}^{\prime} \cdot \Delta x\right) L}{b t^{2} / 6} & =\frac{6 F_{t n} L}{b t^{2}}+\frac{3 E}{L^{3}} \frac{b t^{3}}{12\left(1-\mu^{2}\right)} \frac{6 \Delta x L}{b t^{2}} \\
& =\frac{6 F_{t n} L}{b t^{2}}+\frac{3 E \Delta x}{2\left(1-\mu^{2}\right)} \frac{t}{L^{2}}
\end{aligned}
$$

The maximum stress is taken a derivative with respect to thickness and curve length and the value maximum stress decreases monotonically with thickness and the length in a certain range. Therefore, the parameters of the S-spring should be selected properly to limit the maximum stress to a reasonable range.

\section{A. S-spring Designs of a $150 N$ Propellant Valve}

Regarding to a $150 \mathrm{~N}$ propellant valve, the cross sections of four different types of S-springs are illustrated in Figure IV. The difference between the four sections is that case 1 and case 2 have the different thickness, but the same section structure, and so is the case 3 and case 4 . In addition, case 1 and case 3 have the same thickness, but different section structure, and so is the case 2 and case 4 . Table I lists the design parameters of four S-springs.

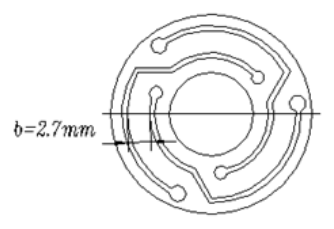

$L=13 \mathrm{~mm} ; b=2.7 \mathrm{~mm} ; t=0.3 \mathrm{~mm} ;$ Case1

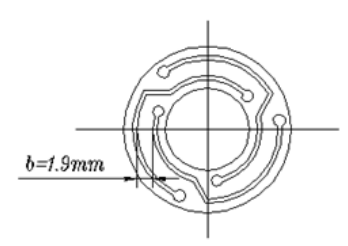

$L=11 \mathrm{~mm} ; b=1.9 \mathrm{~mm} ; t=0.3 \mathrm{~mm} ;$

Case3

FIGURE IV. FOUR DIFFERENT TYPES OF S-SPRING

TABLE I. THE DESIGN PARAMETERS OF FOUR TYPES

\begin{tabular}{|c|c|c|c|c|}
\hline Parameters & Case 1 & Case 2 & Case 3 & Case 4 \\
\hline Bend curve length & $13 \mathrm{~mm}$ & $13 \mathrm{~mm}$ & $11 \mathrm{~mm}$ & $11 \mathrm{~mm}$ \\
\hline Section width & $2.7 \mathrm{~mm}$ & $2.7 \mathrm{~mm}$ & $1.9 \mathrm{~mm}$ & $1.9 \mathrm{~mm}$ \\
\hline Thickness & $0.3 \mathrm{~mm}$ & $0.4 \mathrm{~mm}$ & $0.3 \mathrm{~mm}$ & $0.4 \mathrm{~mm}$ \\
\hline Outer diameter & $24 \mathrm{~mm}$ & $24 \mathrm{~mm}$ & $20 \mathrm{~mm}$ & $20 \mathrm{~mm}$ \\
\hline
\end{tabular}

In the assemble process, a $3 \mathrm{~N}$ preload force should be taken into account to provide the minimum sealing force of the valve. According to the formula (1) (3), the S-spring stiffness of four kinds could be obtained, and consequently the pre-displacement of the spring could be deduced. In order to maintain the same flow resistance for $150 \mathrm{~N}$ thruster valve, the same $0.4 \mathrm{~mm}$ opening distance of the four structures should be guaranteed. Adding up the pre-compression displacement and valve opening distance, the maximum displacement could be obtained, as provided in Table II. Among the four different cases, the maximum stress of case 4 exceeds the unacceptable stress range, meaning that it is easy to fail during repeated moving. With the smallest stress in maximum displacement among the four, the case 2 is the optimist choice for the $150 \mathrm{~N}$ thruster valve.

TABLE II. THE THEORETICAL CALCULATION OF FOUR TYPES

\begin{tabular}{|c|c|c|c|c|}
\hline Parameters & Case 1 & Case 2 & Case 3 & Case 4 \\
\hline Stiffness & $5.47 \mathrm{~N} / \mathrm{mm}$ & $12.97 \mathrm{~N} / \mathrm{mm}$ & $6.35 \mathrm{~N} / \mathrm{mm}$ & $15.06 \mathrm{~N} / \mathrm{mm}$ \\
\hline Pre-compression & $0.548 \mathrm{~mm}$ & $0.231 \mathrm{~mm}$ & $0.472 \mathrm{~mm}$ & $0.199 \mathrm{~mm}$ \\
\hline $\begin{array}{c}\text { Maximum } \\
\text { pressure in } \\
\text { pre-compression }\end{array}$ & $321 \mathrm{MPa}$ & $180.6 \mathrm{MPa}$ & $386 \mathrm{MPa}$ & $217.1 \mathrm{MPa}$ \\
\hline $\begin{array}{c}\text { Maximum } \\
\text { displacement }\end{array}$ & $0.948 \mathrm{~mm}$ & $0.631 \mathrm{~mm}$ & $0.872 \mathrm{~mm}$ & $0.599 \mathrm{~mm}$ \\
\hline $\begin{array}{c}\text { Maximum } \\
\text { pressure in } \\
\text { maximum } \\
\text { displacement }\end{array}$ & $554.8 \mathrm{MPa}$ & $492.3 \mathrm{MPa}$ & $712.7 \mathrm{MPa}$ & $652.8 \mathrm{MPa}$ \\
\hline
\end{tabular}

\section{SUMMARY}

It is the first time to provide a theoretical methodology for the design of S-springs. An optimist S-spring for the $150 \mathrm{~N}$ thruster valve is obtained by using the theoretical method. The future investigations would focus on the numerical simulation and experimental work.

\section{REFERENCES}

[1] Taiichi NAGATA, Kenichi KUSHIKI, Kenichi KAJIWARA, The Development of a Japanese 20N Thruster Valve for a Spacecraft Propulsion System, AIAA, 2006-4876.

[2] G. Schulte, High Performance 400N MMH/NTO Bipropellant Engine for Apogee Boost Maneuvers, AIAA, 99-2466.

[3] Richard L. Huftalen, Next Generation Propellant Isolation Valve Design and Development, AIAA, 2006-4879. 